\title{
Expression Profiling of Genes Associated with Cyanogenesis in Three Cassava Cultivars Containing Varying Levels of Toxic Cyanogens
}

\author{
Morgan Echeverry-Solarte, Victor Ocasio-Ramirez, Annete Figueroa, Eduardo González, \\ Dimuth Siritunga \\ Department of Biology, University of Puerto Rico Mayagüez, Mayagüez, USA. \\ Email: ${ }^{*}$ dimuth.siritunga@upr.edu
}

Received May $4^{\text {th }}, 2013$; revised June $5^{\text {th }}, 2013$; accepted June $30^{\text {th }}, 2013$

Copyright (C) 2013 Morgan Echeverry-Solarte et al. This is an open access article distributed under the Creative Commons Attribution License, which permits unrestricted use, distribution, and reproduction in any medium, provided the original work is properly cited.

\begin{abstract}
Cyanogenic glycosides, linamarin and lotaustralin, are presents in all tissues of Cassava (Manihot esculenta Crantz) except seeds and function as a deterrent for herbivores as well as the translocable form of reduced nitrogen. The genes involved in the cyanogenic pathway [CYP79D1/D2 (EC 1.14.13), linamarase (EC 3.2.1.21), $\alpha$-hydroxynitrile lyase (HNL, acetone-cyanohydrin lyase. EC 4.1.2.37) and $\beta$-cyanoalanine synthase ( $\beta$-CAS. EC 4.4.1.9] have been identified and partially characterized. Our objective was to identify the differential expression pattern of these genes in leaves and roots of three cassava cultivars with varying levels of cyanogenic glucosides. The results show that the differential expression of the genes between leaves and roots is consistent with leaves being the primary site of synthesize of cyanogenic glucosides, which are then translocated to the roots. In addition, the varietal difference for cyanogenic glucoside levels could be explained in part by the combinatorial effort of the synthesis in the leaves and the linamarase catabolic step in the roots. Cluster analysis suggests a coordinated expression between CYP79D1/D2 and $\beta$-CAS genes as well as linamarase and HNL genes, which is in agreement with the spatial separation within a cell of the site of linamarin synthesis (vacuolar) and its breakdown to cyanide (cell wall). Furthermore, cluster analysis for cultivar classification using its gene expression profile match with the reported cyanide levels comparatively for the three cultivars. This is the first study that evaluates the transcriptional activities of the genes involved in the cyanogenic glycoside metabolism using a systematic approach.
\end{abstract}

Keywords: Cassava; Cyanogenic Glucoside; Gene Expression; Real Time PCR

\section{Introduction}

Cassava (Manihot esculenta) is the fourth most important staple food crop in the world after rice, wheat and corn [1]. Cassava roots are consumed daily by more than 500 million people and are the sixth highest source of energy in the world [2]. In agronomical terms, cassava is characterized for its high potential yield, ability to grow in poor soils and drought tolerance [3]. These qualities have made cassava roots an important food crop in the tropics, particularly for subsistence farmers in Africa, who are prompt to suffer droughts and famine.

Cassava has the ability to release hydrogen cyanide (HCN) due to the presence of cyanogenic glycosides

\footnotetext{
"Corresponding author.
}

linamarin ( $>90 \%$ total cyanogen) and lotaustralin $(<10 \%$ total cyanogen) in its tissue $[4,5]$. Cyanogenic glucosides generally function as protective agents against herbivores but more recently has been reported to act as translocable form of reduced nitrogen [4,6,7]. Cyanogenic glucosides are present in all cassava tissue with the exception of seeds, with the leaves having the highest cyanogenic glycoside levels ( $5 \mathrm{~g}$ linamarin $/ \mathrm{kg}$ fresh weight) and the roots having approximately 20 -fold lower linamarin levels [5]. Cyanogenesis in cassava is a complex and variable phenomenon which is influenced by the involvement of different genes and proteins in different tissues, the environment [8] and the developmental stage of the plant [9]. This has lead to the development of a spectrum of cultivars with different levels of cyanogenic glyco- 
sides in the roots. Considering the multiple factor involved in cassava cyanogenesis an explanation for the cultivar-dependent differences in root linamarin content is a controversial aspect in cassava biology [4]. In general, based on cyanogenic glucoside levels all cassava cultivars are categorized into 3 groups: low cyanide $(\leq 50$ $\mathrm{mg}$ linamarin $/ \mathrm{kg}$ fresh weight), intermediate cyanide (50 - $100 \mathrm{mg}$ linamarin/kg fresh weight) and high cyanide ( $\geq 100 \mathrm{mg}$ linamarin/ $\mathrm{kg}$ fresh weight [10].

Cyanogenic glucoside pathway consists of the initial steps in the synthesis of linamarin which then can branch into two pathways depending if the plant is disrupted or if the plant is intact (Figure 1). In the synthesis of cyanogenic glucoside, valine is converted to linamarin (or lotaustralin whose precursor is isoleucine) by the enzymes CYP79D1/D2, CYP71E1 and UDP-glucosyl transferase [11]. Linamarin is stored in the vacuole and does not come in contact with the cell wall bound enzymes, linamarase and hydroxynitrile lyase (HNL), involved in the breakdown process unless the integrity of the cell is compromised [10] usually by a herbivore. Then linamarase can deglucosylate linamarin into acetone cyanohydrin which in turn is decomposed to produce acetone and HCN by HNL $[5,12,13]$. The generation of $\mathrm{HCN}$ from acetone cyanohydrins may also occur at $\mathrm{pH}>5.0$ and temperatures $>35^{\circ} \mathrm{C}$. Overall, in intact plants linamarin is primarily synthesized in the leaves and transported to the roots. Due to the cell wall localization of linamarin metabolizing enzymes, an apoplastic mode of linamarin transportation has been suggested. Linustatin, the glycosylated form of linamarin, is thought to be the translocable form, since its presence in other

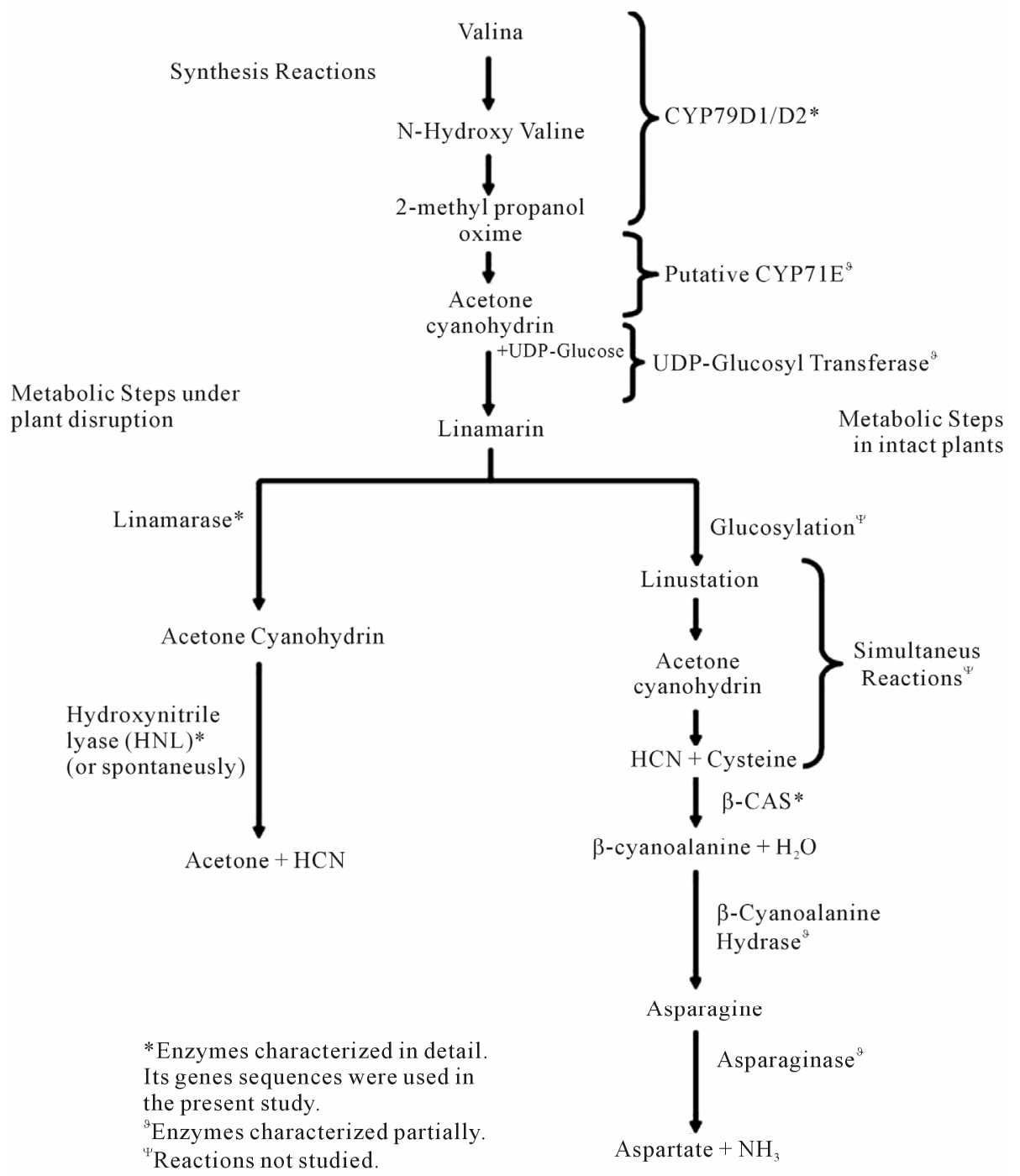

Figure 1. Cyanogenic glycosides metabolic pathways. Three main sets of reaction are illustrated: 1) the synthesis; 2) the metabolic steps under plant disruption and; 3) the metabolic steps in intact plants. All the reactions have not been studied in detail with most of them being inferred from research in Hevea brasilensis and Sorgum bicolor. 
cyanogenic plants has been demonstrated [14-16].

Further support for leaves being the primary site of linamarin synthesis in cassava is derived from the silencing of CYP79D1/D2 genes selectively in leaves of transgenic plants. This resulted in the significant reduction in both leaf and root linamarin content, while a root specific silencing of CYP79D1/D2 led to no change in linamarin accumulation in any tissue [7,17]. Siritunga et al. [17] has shown that linamarin is a mobile source of reduced nitrogen $\left(\mathrm{NH}_{3}\right)$ to cassava root. Thus, the linamarin is metabolized in differently in intact plants. Selmar [16] by extrapolating the results and evidence found in Hevea brasilensis, suggested that once in the roots, linustatin can be deglucosilated in two different manners by "sequential" and "simultaneous" cleavage. In the sequential cleavage the linustatin is converted back to linamarin and possibly stored, but in the simultaneous reaction the linustatin is converted in acetone-cyanohydrin, which can produce free $\mathrm{HCN}$ at $\mathrm{pH}>5.0$ and temperatures $>35^{\circ} \mathrm{C}$. Therefore the plant needs to re-assimilate the $\mathrm{HCN}$ through detoxification reactions to avoid autotoxicity within its cells. Nartey [18] was the first to demonstrate the presence of such a detoxification pathway in cassava when $49 \%$ of the ${ }^{14} \mathrm{C}$-radiolabeled cyanide fed to leaves accumulated as asparagine in two week old cassava seedlings, thus proposing a cyanogen re-assimilation pathway in cassava. Subsequent studies have shown that re-assimilation of cyanide to asparagine occurs through an intermediate, $\beta$-cyanoalanine [18-20], in reactions catalyzed by $\beta$-cyanoalanine synthase and $\beta$ cyanoalanine hydrase [21].

The contribution of each gene involved in the cyanogenic glucoside synthesis, breakdown and re-assimilation pathway to the total cyanogenic glucoside content is unknown. The previous transcriptional studies have focused on a particular gene under a specific condition $[6,7,8,17]$ and never has there been an integral study comparing the activity of the key genes of the cyanogenic glocuside patherway together (Table 1). Here we present the complete tissue-specific expression analysis of key genes in the cyanogenic glucoside pathway in three agronomically important cassava cultivars of varying cyanogen levels (Mcol2215-low level, 60444-mid level, Mtai16-high level) in order to better understand the contribution of each gene to the different cyanogenic glucoside content of cultivars.

\section{Methods}

\subsection{Plant Material and Growth Conditions}

Three cassava cultivars with varying levels of cyanogens were used: Mcol2215 (sweet cultivar), 60444 (bittersweet cultivar) and Mtail6 (bitter cultivar). Eight month old roots of Mcol2215, 60444 and Mtai16 have 142, 182 and 569 ppm total cyanogens per gram dry weight, respectively (Teresa Sanchez, CIAT, personal communication). All plants were grown under in vitro conditions in $4 \mathrm{E}$ liquid media [17] using $14 \mathrm{~h}$ /day photoperiod (5 $\mu \mathrm{mol}$ photons $\mathrm{m}^{-2} \cdot \mathrm{s}^{-1}$ ) at $28^{\circ} \mathrm{C}$.

\subsection{RNA Isolation and cDNA Synthesis}

Leaves and roots from 4 month old plants were collected from in vitro plants, frozen immediately in liquid nitrogen and stored at $-80^{\circ} \mathrm{C}$ until use. RNA isolations were performed using RNeasy ${ }^{\circledR}$ Mini Kit followed by the elimination of DNA contaminants by RNAse-Free DNAse treatment according to manufacturers' recommendations (Qiagen Inc, Valencia, CA, USA). After analysis of quality in a $0.8 \%$ agarose gel and quantification, the RNA was protected with 1 unit of RNasin ${ }^{\circledR} \mathrm{Ri}$ bonuclease inhibitor (Promega Inc, Madison, WI, USA). The cDNA was synthesized from $1 \mu \mathrm{g}$ of total RNA using Omniscript RT $^{\circledR}$ kit (Qiagen Inc, Valencia, CA, USA) with $1 \mu \mathrm{M}$ of Oligo (dT) $)_{12-18}$ and $12.5 \mathrm{ng} / \mu \mathrm{L}$ of random primers. The mixture was incubated for 2 hours at $37^{\circ} \mathrm{C}$.

\subsection{Primers Design}

The genes studied were CYP79D1/D2, linamarase, HNL, $\beta$-CAS and $18 \mathrm{~s}$ rRNA (reference gene). Due to the $85 \%$ identity between CYP79D1 and CYP79D2 primers were

Table 1. Summary of previous studies on the expression of key genes involved in cyanogenic glycoside metabolism in cassava.

\begin{tabular}{|c|c|c|c|c|}
\hline \multirow{2}{*}{ Gene } & \multirow{2}{*}{ Reference } & \multirow{2}{*}{ Technique } & \multicolumn{2}{|c|}{ Detection } \\
\hline & & & Leaves & Roots \\
\hline \multirow{2}{*}{ CYP79D1/D2 } & Siritunga and Sayre, 2003 [17] & Retrotrasncriptase PCR Semiquantitative & Yes & Yes \\
\hline & Jørgensen et al., 2005 [6] & In Tube in Situ Retrotranscription PCR on Tissue Sections & Yes & Not attempted \\
\hline Linamarase & Santana et al., 2002 [9] & Retrotrasncriptase PCR Semiquantitative & Yes & Not Detection \\
\hline HNL & White et al., 1998 [5] & Northern blot & Yes & Yes \\
\hline$\beta$-CAS & No references yet & Not attempted & Not attempted & Not attempted \\
\hline
\end{tabular}


designed for consensus regions on both genes [11]. Each primer (Table 2) was designed using Primer Quest ${ }^{S M}$ software from IDT (Integrated DNA Technologies Inc, Coralville, IA, USA) and was checked for their compliance to the recommendations of the ABI guidelines for Real-Time PCR Primers and amplicons (Real-Time PCR handbook, University of Illinois, Urbana-Champagne, IL, USA). Subsequently, the primers generated and the original GenBank sequences were analyzed using BLAST program to verify the absence of homologies between the primers and other reported cassava sequences.

\subsection{Conventional PCR Conditions}

Conventional PCR was performed with $10 \mu \mathrm{L}$ from a 1:8 cDNA dilution. For the genes CYP79D1/D2, linamarase and $\beta$-CAS the reactions contains $1 \times$ of PCR Buffer, 2.5 $\mathrm{mM}$ of $\mathrm{MgCl}_{2}, 0.4 \mu \mathrm{M}$ from each gene-specific primer, $0.2 \mathrm{mM}$ of dNTPs and 1 unit of Taq polymerase in a final volume of $20 \mu \mathrm{L}$. For amplification of HNL and 18s rRNA the reaction conditions were similar, except for the concentration of the gene-specific primers were $0.2 \mu \mathrm{M}$. The PCR conditions used were an initial denaturation at $96^{\circ} \mathrm{C}$ for 5 minutes followed by 35 cycles of $96^{\circ} \mathrm{C}$ for 1 minute, specific annealing temperature for each primer (CYP79D: $60^{\circ} \mathrm{C}$, linamarase: $60^{\circ} \mathrm{C}$; $\mathrm{HNL}: 58^{\circ} \mathrm{C} ; \beta$-CAS: $56^{\circ} \mathrm{C}$ and $18 \mathrm{~s}$ rRNA: $55^{\circ} \mathrm{C}$ ) for 1 minute and an extension at $72^{\circ} \mathrm{C}$ for 1 minutes. Final extension at $72^{\circ} \mathrm{C}$ for 5 minutes was also performed for all reactions. All the reactions were run in an Eppendorf Mastercycler ${ }^{\circledR}$ thermocycler (Eppendorf, Hamburg, Germany).

\subsection{Quantitative Real Time PCR Conditions}

All real time PCR amplifications were performed with 2 $\mu \mathrm{L}$ from a 1:8 cDNA dilution and in a final volume of 20 $\mu \mathrm{L}$ using DyNAmo SYBR $^{\circledR}$ Green qPCR kit (Finnzymes Keilaranta, Finland) and $1 \times$ of ROX $^{\circledR}$ (Invitrogen Inc, Carlsband, CA, USA) as internal reference. Varied genespecific primer concentrations were used $(0.05 \mu \mathrm{M}$ of each primer for CYP79D and $\beta$-CAS amplification, 0.1 $\mu \mathrm{M}$ of each primer for linamarase and HNL amplification and $0.15 \mu \mathrm{M}$ of each primer for 18s rRNA amplification). Three technical repetitions (of each sample) were performed. All reactions were conducted in an ABI7300 real-time PCR thermocycler (Applied Biosystems, Foster City, CA, USA) which automatically calculated the dissociation curve. The amplification consisted of an initial denaturation at $94^{\circ} \mathrm{C}$ for 5 minutes followed by 30 cycles of $94^{\circ} \mathrm{C}$ for 20 seconds (for CYP79D and linamarase) or for 15 seconds (for HNL, $\beta$-CAS and 18s rRNA), annealing at $58^{\circ} \mathrm{C}$ for 1.25 minutes (for CYP79D1/D2) or $62^{\circ} \mathrm{C}$ for 1 minute (for linamarase, HNL and $\beta$-CAS) or $53^{\circ} \mathrm{C}$ for 35 seconds (for $18 \mathrm{~s}$ rRNA).

\subsection{Data Acquisition}

The quality of amplification from each real-time PCR reaction was assessed through the dissociation curves generated by the SDS Software (version 1.3.1) incorporated to the ABI7300 real-time PCR thermocycler. The $C t$ data were collected using the manual threshold method which posts the threshold in logarithmic phase.

Table 2. Genes studied and their respective primer sequences utilized.

\begin{tabular}{|c|c|c|c|c|}
\hline Gene & & Primer Sequence & Gen Bank Accession Number & Amplicon Size (bp) \\
\hline \multirow{2}{*}{ CYP79D1/D2 } & Forward & 5'-CTCCATGGCCACTCATCGGAAA-3' & \multirow{2}{*}{$\begin{array}{l}\text { AF140613 } \\
\text { AF140614 }\end{array}$} & \multirow{2}{*}{86} \\
\hline & Reverse & 5'-TGTCCTTCATGAGTTGGTGAATCC-3' & & \\
\hline \multirow{2}{*}{ Linamarase (A) } & Forward & 5'-CTCAAGCACTGCAGGACAAA-3' & \multirow{2}{*}{ S35175 } & \multirow{2}{*}{158} \\
\hline & Reverse & 5'-ATCATGGGCAAATCCAACAT-3 & & \\
\hline \multirow{2}{*}{ Linamarase (B) } & Forward & 5'-CCTAACAAGGTATCCCAAGAAATCG-3' & \multirow{2}{*}{ S35175 } & \multirow{2}{*}{111} \\
\hline & Reverse & 5'-CCAACCTTCCTTGAATCCTTTGATG-3' & & \\
\hline \multirow[b]{2}{*}{ HNL } & Forward & 5'-GCTTTTGGAGTCGTTTCCTG-3' & \multirow[b]{2}{*}{ Z29091 } & \multirow[b]{2}{*}{228} \\
\hline & Reverse & 5'-CCGTAACCTTTTTCGGTGAA-3' & & \\
\hline \multirow{2}{*}{$\beta$-CAS } & Forward & 5'-TCCACTGCGAGAGAGAGCTTAGAG-3' & \multirow{2}{*}{ EU350583 } & \multirow{2}{*}{99} \\
\hline & Reverse & 5'-GCCATTGATGTAAGAGTAGCCATTG-3' & & \\
\hline \multirow{2}{*}{18 s rRNA } & Forward & 5'-ATGATAACTCGACGGATCGC-3 & \multirow{2}{*}{ AB233568 } & \multirow{2}{*}{169} \\
\hline & Reverse & 5'-CTTGGATGTGGTAGCCGTTT-3' & & \\
\hline
\end{tabular}


Amplifications with primer-dimmer or non-amplifications were eliminated from the analysis or replaced with cycle number corresponding to the Limit of Detection + 1 cycle (LOD +1 cycle) in order to correct for off-scale measurements. The LOD is the highest CT which is obtained for a truly positive sample, thus the LOD +1 corresponds to $50 \%$ of the amount that you are able to detect (Mikael Kubista, personal communication).

The following criteria were followed for acquisition of data: 1) if the three technical repetitions amplified clearly, no data was removed or replaced. The $C t$ for the biological sample was the average from these three technical repetitions; 2) if one technical repetition amplified with some problem it was eliminated from the data. The $C t$ for the biological sample was the average from the two remaining technical repetitions; 3 ) if two technical repetitions were not reliable, these were eliminated from the data and the $C t$ for the remaining replicate was reported for the corresponding biological sample; 4) if none of the three technical repetitions amplified then it was considered that the sample has very low transcriptional activity and the data is replaced with the LOD +1 cycle. In this experiment the LOD was calculated for the genes CYP79D1/D2 and linamarase using dilutions of amplicons purified.

\subsection{Data Pre-Treatment and Statistical Analysis}

Two types of univariable comparison for the transcriptional activity for each gene were developed: 1) Comparison between tissues (leaves vs roots) in each cultivar; 2) Comparisons among the three cultivars for each tissue. The $C t$ data of all the genes were formatted in different matrices depending on the comparisons being conducted. The matrices were $n \times 5$ type where the 5 refers to the number of genes studied (CYP79D1/D2, linamarase, HNL, $\beta$-CAS and 18s) and $n$ refers to the number of samples being compared, depending on the corresponding biological replicates (3) and technical replicates (3). Thus, in the comparison between tissues matrices $18 \times 5$ were obtained, whereas on the comparison among cultivars (in each tissue separated) matrices $27 \times 5$ were used. The matrices were introduced into GenEx Light ${ }^{\mathbb{B}}$ software following the next data pre-processing which are correction for primer efficiency (calculated from standard curves), technical replicates average, correction for reference gene (18s), production of relative quantities for the average of $\mathrm{Ct}$ followed by the transformation of data to fold changes using Logarithmic in base 2 (all data preprocessing were conducted using equations described in $\mathrm{GenEx}^{\circledR}$ manual). The GenEx-processed data from each matrix were used for statistical analysis according to the corresponding experimental design. In tissue comparison (leaves vs Roots) a $t$-paired test was conducted while for the comparison between cultivars an ANOVA for completely randomized design and contrast was conducted. The statistical significance used was $10 \%$. The confidence intervals for each mean and for the mean difference were made using $1-\alpha=90 \%$.

The overall comparison among the genes as well as the cluster analysis were developed using a multivariable approach. It was a matrix of $57 \times 5$ that included the $C t$ data from the three cultivars (including the three biological replicates), the two tissues and an artificial sample with its respective technical replicates (3). The artificial sample is a mixture of the target amplicons purified, which are at the same concentration. The matrix was introduced into GenEx Light ${ }^{\mathbb{B}}$ software and followed the same data pre-processing described above, but the relative quantities were obtained with respect to the $C t$ averages from the artificial sample. These GenEx processed data was used for chart bar visualization of all the genes in the three cultivars in both tissues. For Gene expression clusters according to each cultivar, the data processed from this matrix was separated according to the cultivar. The data from leaves and roots were analyzed together as one vector and thus was not distinguished. For cultivar clusters according to the transcriptional activity of all the genes was used the full data released after GenEx pre-processing. In both cases the clusters were generated with Infostat ${ }^{\mathbb{B}}$ for non-standardized data using Euclidean distance and unweighted centroid method.

\section{Results}

\subsection{Comparison of Quantitative Gene Expression within Tissues}

In all three cultivars analyzed the expressions of CYP79D1/D2, linamarase and HNL genes were higher in the leaves than the roots (Figure 2; Table 3). In the cases of linamarase and HNL the lowest leaves/roots expression ratio was observed for the low cyanide cultivar Mcol2215, 38.86 and 178.52, respectively. Though melting curves for amplicons were detected for CYP79D1/D2 activity in cassava roots from the three cultivars, its detection was intermittent. The leaves/roots expression ratio of CYP79D1/D2 genes showed a pattern with lowest ratio being in the low cyanogen cultivar and the highest ratio being in the high cyanogen cultivar $($ Mcol2215 $=4.44,60444=16.44$ and Mthai16 $=354.78)$ (Table 3). The expression of $\beta$-CAS gene also showed a pattern with the low cyanogen cultivar Mcol2215 having a higher root expression compared to expression in the leaf (roots/leaves ratio $=4.89)$. This ratio reduced in the intermediate cyanogen cultivar 60444 to 2.86 with the 

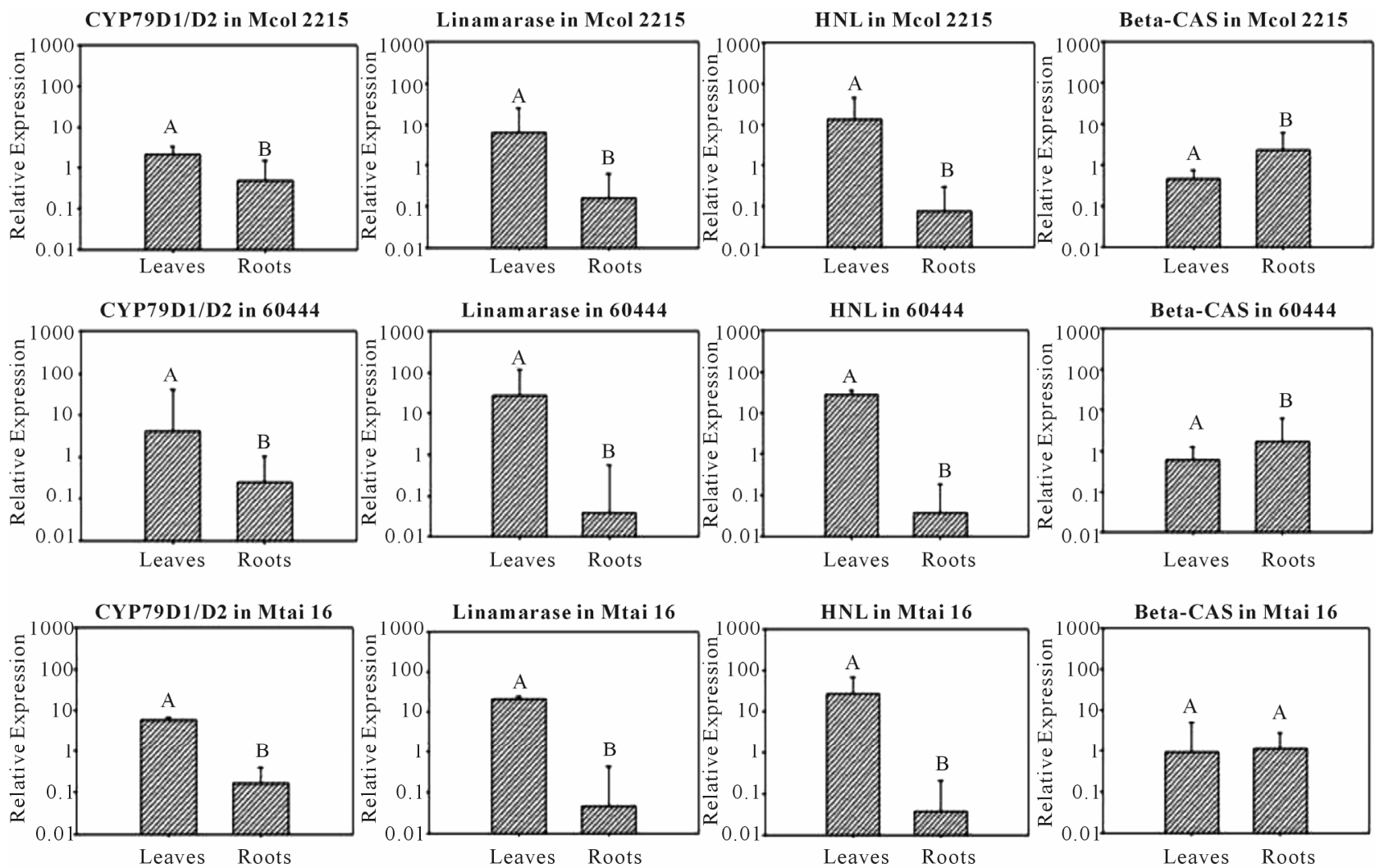

Figure 2. Comparison between leaves and roots of the transcriptional activity of the genes CYP79D1/D2, linamarase, HNL and $\beta$-CAS in three cassava cultivars. The genes CYP79D1/D2, linamarase and HNL have highest transcriptional activity in leaves in relation to roots while $\beta$-CAS gene has more transcriptional activity in roots or at least the same level in both tissues. The error bar is the upper value of $\mathbf{9 0 \%}$ confidence interval. A different letter above the bars indicates significant differences $(\mathbf{p} \leq \mathbf{0 . 1 0})$.

Table 3. Ratios of gene expression between leaves and roots in the three cassava cultivars.

\begin{tabular}{cclccc}
\hline Cultivar & Gene & Comparison & Ratio & $\begin{array}{c}\text { Two sided C. I for means } \\
\text { differences }(\alpha=10 \%)\end{array}$ & p-value (1-side) \\
\hline Mcol 2215 & CYP79D1/D2 & Leaves/Roots & 4.44 & $(1.28 ; 15.45)$ & $0.0364^{*}$ (Right) \\
Mcol 2215 & Linamarase & Leaves/Roots & 38.86 & $(2.7 ; 556.41)$ & $0.0284^{*}$ (Right) \\
Mcol 2215 & HNL & Leaves/Roots & 178.52 & $(58.49 ; 548.75)$ & $0.0027^{*}$ (Right) \\
Mcol 2215 & $\beta$-CAS & Roots/Leaves & 4.89 & $(1.15,20.83)$ & $0.0428^{*}$ (Right) \\
60444 & CYP79D1/D2 & Leaves/Roots & 16.44 & $(1.26 ; 215.27)$ & $0.0431^{*}$ (Left) \\
60444 & Linamarase & Leaves/Roots & 724.01 & $(106.89 ; 4904.87)$ & $0.0049^{*}$ (Right) \\
60444 & HNL & Leaves/Roots & 719.29 & $(177.29 ; 1176.23)$ & $0.0026^{*}$ (Right) \\
60444 & $\beta$-CAS & Roots/Leaves & 2.86 & $(0.86 ; 9.58)$ & $0.0624^{*}$ (Left) \\
Mthai 16 & CYP79D1/D2 & Leaves/Roots & 34.78 & $(14.03 ; 86.22)$ & $0.0038^{*}$ (Right) \\
Mthai 16 & Linamarase & Leaves/Roots & 458.26 & $(41.93 ; 5007.9)$ & $0.0087^{*}$ (Right) \\
Mthai 16 & HNL & Leaves/Roots & 699.41 & $(112.98 ; 4359.6)$ & $0.0045^{*}$ (Right) \\
Mthai 16 & $\beta$-CAS & Leaves/Roots & 1.12 & $(0.52 ; 2.86)$ & 0.2883 (Left) \\
\hline
\end{tabular}

*Indicates significant difference. 
high cyanogen cultivar Mthai16 having a leaves/roots ratio of 1.12. Out of the four gene expressions analyzed in this study only $\beta$-CAS gene was shown to be having an equal or higher expression level in the roots compared to leaves (Figure 2; Table 3).

\subsection{Comparison of Quantitative Gene Expression within Cassava Cultivars}

In leaves the average transcriptional activity of all the genes with exception of HNL has a pattern directly related to the cyanogens levels reported for each cultivar (Figure 3; Table 4). In each case, the expression levels in Mtai16 leaves are statistical different $(1-\alpha=10 \%)$ from Mcol2215, as well as from the cultivar 60444 in CYP79D1/D2, HNL and $\beta$-CAS genes. However, no significant differences were found in any case between Mcol2215 and 60444 or between Mtai 16 and 60444 for linamarase gene. When comparing the leaf expression ratio for each gene between any two cultivars, 60444: Mcol215 ratios were the lowest with CYP79D1/D2, linamarase, HNL and $\beta$-CAS being $2.82,1.75,1.34$ and 1.67 respectively (Table 4 ). In roots the average transcriptional activity of any of the genes did not show any pattern based on the cyanogen content of the cultivars. Though no pattern was observed, statistically significant differences in the gene expression were observed for

Table 4. Ratios of expression among cassava cultivars with different cyanide levels.

\begin{tabular}{|c|c|c|c|c|c|}
\hline Tissue & Gene & Comparison & $\begin{array}{c}\text { Means } \\
\text { Differences: Ratio }\end{array}$ & $\begin{array}{l}\text { Two sides C. I for means } \\
\text { differences }(\alpha=10 \%)\end{array}$ & Contrast. p-value \\
\hline Leaves & CYP79D1/D2 & Mthai16/Mcol2215 & 13 & $(3.66 ; 46.21)$ & $0.0077^{*}$ \\
\hline Leaves & CYP79D1/D2 & Mthai16/TMS60444 & 4.63 & $(1.31 ; 16.56)$ & $0.0571^{*}$ \\
\hline Leaves & CYP79D1/D2 & TMS60444/Mcol2215 & 2.82 & $(0.80 ; 10.05)$ & 0.1636 \\
\hline Leaves & Linamarase & Mthai16/Mcol2215 & 3.41 & $(1.13 ; 10.26)$ & $0.0737^{*}$ \\
\hline Leaves & Linamarase & Mthai16/TMS60444 & 1.95 & $(0.65 ; 5.86)$ & 0.2857 \\
\hline Leaves & Linamarase & TMS60444/Mcol2215 & 1.75 & $(0.58 ; 5.28)$ & 0.3594 \\
\hline Leaves & HNL & Mthai16/Mcol2215 & 5.35 & $(2.27 ; 12.64)$ & $0.0092^{*}$ \\
\hline Leaves & HNL & Mthai16/TMS60444 & 7.16 & $(3.03 ; 16.91)$ & $0.0044^{*}$ \\
\hline Leaves & HNL & Mcol2215/TMS60444 & 1.34 & $(0.56 ; 3.16)$ & 0.5332 \\
\hline Leaves & $\beta$-CAS & Mthai16/Mcol2215 & 6.27 & $(2.23 ; 17.63)$ & $0.0134^{*}$ \\
\hline Leaves & $\beta$-CAS & Mthai16/TMS60444 & 3.76 & $(1.34 ; 10.56)$ & $0.0473^{*}$ \\
\hline Leaves & $\beta$-CAS & TMS60444/Mcol2215 & 1.67 & $(3.66 ; 46.21)$ & 0.3676 \\
\hline Roots & CYP79D1/D2 & Mtai16/Mcol2215 & 1.67 & $(1.16 ; 5.50)$ & 0.4367 \\
\hline Roots & CYP79D1/D2 & Mtai16/TMS60444 & 2.18 & $(0.66 ; 7.21)$ & 0.2488 \\
\hline Roots & CYP79D1/D2 & Mcol2215/TMS60444 & 1.31 & $(0.39 ; 4.31)$ & 0.6727 \\
\hline Roots & Linamarase & Mcol2215/Mtai16 & 3.44 & $(0.45 ; 26.17)$ & 0.283 \\
\hline Roots & Linamarase & Mtai16/TMS60444 & 3.1 & $(0.40 ; 23.59)$ & 0.3235 \\
\hline Roots & Linamarase & Mcol2215/TMS60444 & 11.1 & $(1.40 ; 81)$ & $0.0650^{*}$ \\
\hline Roots & HNL & Mtai16/Mcol2215 & 1.36 & $(0.31 ; 5.90)$ & 0.6936 \\
\hline Roots & HNL & Mtai16/TMS60444 & 7.36 & $(0.58 ; 31.78)$ & $0.0381^{*}$ \\
\hline Roots & HNL & Mcol2215/TMS60444 & 5.55 & $(1.24 ; 23.26)$ & $0.0668^{*}$ \\
\hline Roots & $\beta$-CAS & Mtai16/Mcol2215 & 1.56 & $(0.55 ; 4.35)$ & 0.4326 \\
\hline Roots & $\beta$-CAS & Mtai16/TMS60444 & 1.57 & $(0.56 ; 4.38)$ & 0.421 \\
\hline Roots & $\beta$-CAS & TMS60444/Mcol2215 & 1 & $(0.36 ; 2.80)$ & 0.9827 \\
\hline
\end{tabular}

*Indicates significant difference. 

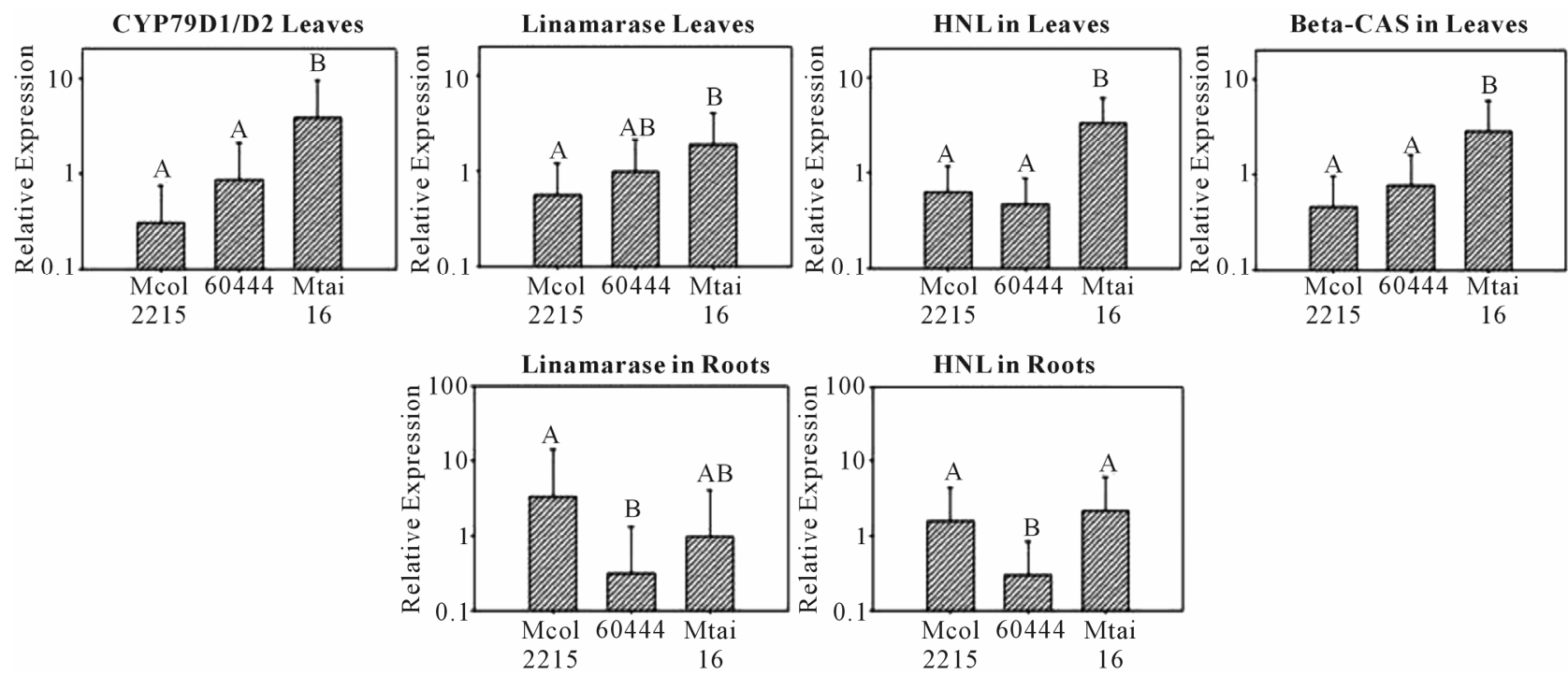

Figure 3. Expression pattern of the key genes involved in cyanogenic glycoside metabolism in leaves and roots of three cassava cultivars with different cyanide level. Only illustrated are the gene expression pattern where significant differences were found. The cultivars used were Mcol2215 (142 ppm of HCN), 60444 (182 ppm of HCN) and Mtai16 (569 ppm of HCN). The error bar is the upper value of $90 \%$ confidence interval. A different letter above the bars indicates significant differences $(p \leq$ 0.10).

linamarase and HNL (Figure 3). The expression of linamarase and HNL were significantly different between Mcol2215 and TMS6044. Gene expression of CYP79D1/ $\mathrm{D} 2$ and $\beta$-CAS in the roots showed no differences between cultivars. Overall, greater differences in gene expression between cultivars were observed in the leaves and not the roots (Table 4 ).

\subsection{Overall Gene Expression Comparison}

Figure 4 illustrates a general and comprehensive comparison of the expression levels of the four genes in the leaves and roots of three cultivars having varying levels of cyanogens. With the exception of $\beta$-CAS all genes are expressed higher in the leaves compared to the roots. The expression level of $\beta$-CAS remained constant between leaves and roots in the three cultivars analyzed. Overall HNL had the highest expression in each of the cultivars irrespective of the tissue while the lowest levels were observed for the expression of CYP79D1/D2 genes.

\subsection{Cluster Analysis}

In all three cultivars studied here, based on expression of the four genes collectively, CYP79D1/D2 (cyanogen synthesis) and $\beta$-CAS (cyanogen re-assimilating) clustered together (Figure 5). Linamarase and HNL genes, both involved in the breakdown of cyanogens upon tissue disruption, based on their expression clustered together only in Mcol2215 and 60444 cultivars (Figures 5(a) and (b)). In Mtai16, linamarase showed a closer relationship with CYP79D1/D2 and $\beta$-CAS expression than the expression of HNL (Figure 5(c)). In other hand, the cultivar cluster, which is also based in the expression of the four genes together, shows that cultivars Mcol2215 and 60444 were more related with each other than with Mtai16 (Figure 6).

\section{Discussion}

For years, the metabolism of cyanogenic glycosides in cassava has been studied due to the agronomical importance of this crop. The initial studies showed that cyanogens have herbivore deterrent abilities, however, more recently an alternate purpose of serving as mobile nitrogen storage source has been proposed. These studies have shown that these molecules are synthesized in leaves and translocated to roots, where they are utilized for amino acid synthesis [6,7]. Nevertheless, to date many aspects such as the regulation of the genes involved with cyanogens as well as details about their transport from leaves to roots remain unknown [16]. The present study demonstrates for the first time a systematic and quantitative analysis of the transcriptional activity of the genes involved in the metabolism of cyanogenic glycosides using three different cassava cultivars, each having varying levels of cyanogens.

The results through conventional PCR or real time PCR marginally differ from the previous studies conducted for the expression of CYP79D1/D2 genes, linamarase gene and HNL gene (Table 1). Siritunga et al. [17] showed transcriptional activity of CYP79D1/D2 in 


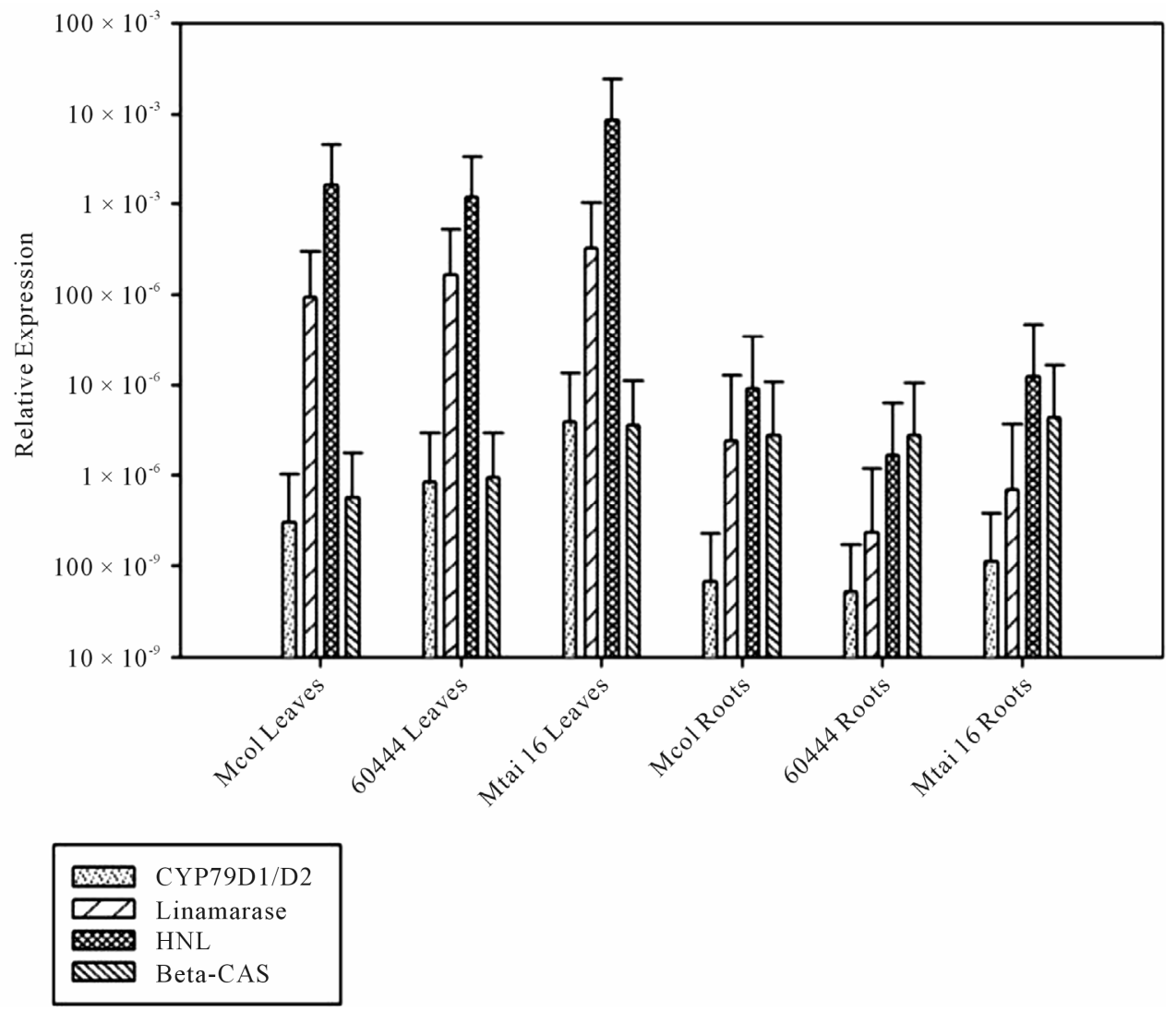

Figure 4. Gene Expression profiles of four genes involved in cyanogenic glycosides pathway in leaves and roots of three cassava cultivars. The cultivars used were Mcol2215 (142 ppm of HCN), 60444 (182 ppm of $\mathrm{HCN})$ and Mtai16 (569 ppm of HCN).The leaves have the higher transcriptional activity for the genes CYP79D1/D2, linamarase and HNL as compared to the roots, whereas $\beta$-CAS gene has higher transcriptional activity in roots than in leaves or at least the same level in both tissues. When the cultivars are compared, the pattern illustrated in CYP79D1/D2 genes in leaves could explain the cyanide differences, as well as the pattern found in the linamarase in roots. The error bar is the upper value of $90 \%$ confidence interval. A different letter above the bars indicates significant differences $(\mathrm{p} \leq 0.10)$.

Cultivar Mcol 2215

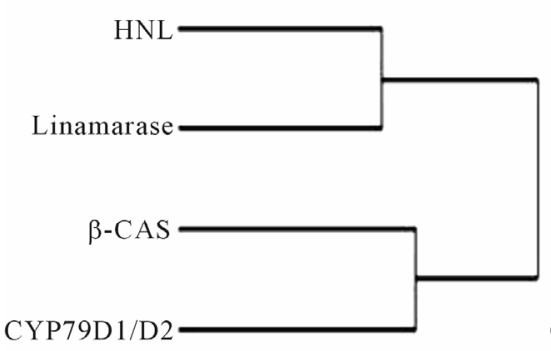

Cultivar 60444

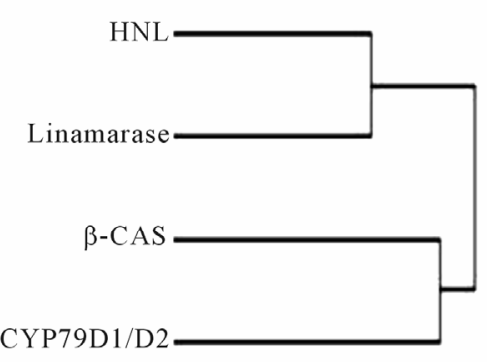

Cultivar Mtai 16

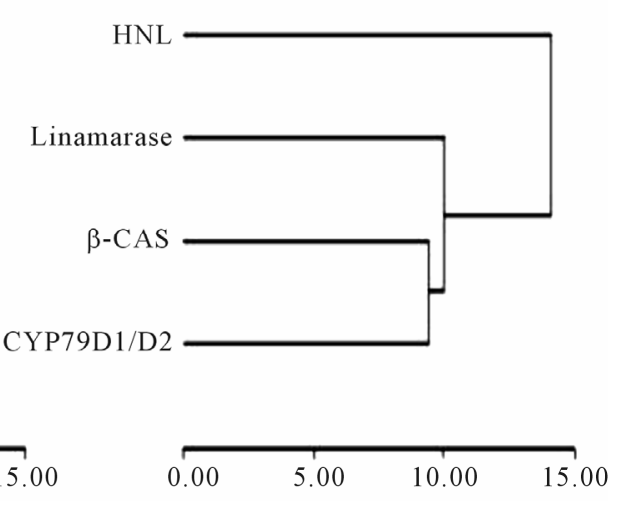

(c)

(a)

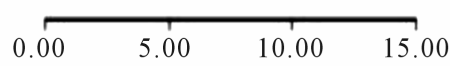

(b)

Figure 5. Dendograms from cluster classification of the genes involved in cyanogenic glycoside metabolism in three cassava cultivars. The data from leaves and roots were analyzed together. Two clusters were generated in Mcol2215 and 60444: 1) Synthesis/Re-assimilation cluster (CYP79D/Linamarse). 2) Catabolic cluster (linamarase/HNL). Meanwhile in Mtai16 only the Synthesis/Re-assimilation cluster was observed. In Mtai16, HNL could be classified separately due to the higher level of transcriptional activity in this cultivar. On the other hand, this result agrees with the cyanide levels in the three cultivars: Mcol2215 (142 ppm of HCN), 60444 (182 ppm of HCN) and Mtai16 (569 ppm of HCN). 


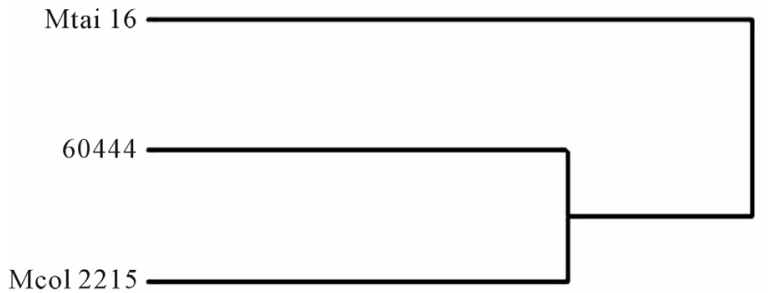

$\begin{array}{llll}0.00 & 1.00 & 2.00 & 3.00\end{array}$

Figure 6. Dendograms from cluster analysis of three cassava cultivars with different cyanide. The data from leaves and roots were analyzed together. The cluster generated match with the cyanide similarities among the three cultivars [Mcol2215 (142 ppm of HCN), 60444 (182 ppm of HCN) and Mtai16 (569 ppm of $\mathrm{HCN})]$.

roots, while in this study conventional PCR and real time PCR were able to detect expression of CYP79D1/D2 in the three cultivars intermittently. Similarly Santana et al. [9] reported that the expression of linamarase gene in the roots was not detected, leading to the prediction that linamarase enzyme is transported to the roots from the leaves. In our study the expression of linamarase was detected in roots in all three cultivars, though at a lower rate that in the leaves, thus not discounting the hypothesis that linamarase in transported. In the case of HNL, at difference of White et al. [5], we observed transcriptional activity of this gene in the roots. Nevertheless those authors also reported the scarce enzymatic activity of this protein in the roots, which allow us to suggest the presence of a post-transcriptional regulation mechanism for the expression of the HNL enzyme in cassava. It is important to state that none of these contrasting results disprove the hypothesis that linamarin translocation occurs in cassava, likely as linustatin [6,16,17,22,23]. Overall, the differences in the expression of these genes between various studies could lie in the use of plants at different development stages, use of different media components and growth conditions as well as technical differences such as different RNA isolation, cDNA synthesis and PCR conditions. In this study though, real-time PCR technique was used followed by dissociation curve analysis making this analysis more sensitive and equipped with a wider dynamic range than conventional PCR [24, 25]. In conclusion, the expression of all 4 genes was observed in all 3 cultivars, but at very low-level for the expression of CYP79D1/D2 and linamarase in roots.

In the comparisons between tissues the results are accord with previous research work $[9,10,19,22]$, so that all the genes with exception of $\beta$-CAS have more transcriptional activity in leaves that in roots (Figure 2; Table 3).
The high CYP79D1/D2 levels in leaves, as well as the high $\beta$-CAS levels in the roots, match with the evidences that suggest that cyanogenic glycosides are synthesized in leaves and afterward translocated to the roots, where are re-assimilated $[14,16,24,25]$. The high presence of CYP79D1/D2, linamarase and HNL transcripts in leaves in relation to the roots could be associated with defense mechanisms against herbivores. In this way, the plant accumulates high levels of linamarase and HNL in its leaves, which can insure the degradation of linamarin under herbivores attacks. The evidence found in the comparison among cultivars on the leaves, as well as the cluster analysis, suggest the importance of the accumulation of these enzymes and its role in the plant defense (see below). On the other hand the higher expression levels of $\beta$-CAS in the roots results in the higher root $\beta$-CAS enzyme activity ( $36 \mu \mathrm{g} \mathrm{H} 2 \mathrm{~S} / \mathrm{min} / 100 \mathrm{mg}$ protein) demonstrated by Elias et al. [26,27] compared to leaves (13 $\mu \mathrm{g} \mathrm{H} 2 \mathrm{~S} / \mathrm{min} / 100 \mathrm{mg}$ protein). Since the conversion of cyanide to non-protein amino acid $\beta$-cyanoalanine, through a condensation reaction with cysteine, catalyzed by $\beta$-cyanoalanine synthase (Figure 1), is thought to be the major cyanide detoxification pathway in cassava, the presence of elevated levels of $\beta$-CAS enzyme prevents the build up of HCN to toxic levels in the cells.

A comprehensive understanding of the differences in the levels of cyanogenic glycosides among cassava cultivars have been one of the more challenging research topic in this plant [17]. In order to understand those cultivar differences, we can infer that the synthesis of cyanogenic glycoside is higher in a high-cyanide cultivar in relationship with a low-cyanide cultivar. Our results in the comparison among leaves are according with this hypothesis. Thus, we observed the presence of comparatively higher levels of CYP79D1/D2 expression in leaves of the bitter cassava Mtai16 compared to TMS60444 and Mcol2215 which have lower cyanogen levels (Figure 3; Table 4). Nevertheless in the roots we did not observed differences among the cultivars in the expression of CYP79D1/D2, which reinforces the importance of the translocation. Thus, the conversion of valine to 2-methyl propanol oxime in the leaves might be the controlling point in the synthesis of linamarin in cassava and an important step that determine the cultivars differences. On the other hand, for the genes involved in the catabolism, the expression of linamarase and HNL would have thought to be lower in the bitter Mtail6 cultivar permitting the increase accumulation of the toxic cyanogens. However it was observed that Mtai16 had the highest expression of linamarase and HNL in the leaves compared to the other cultivars (Figure 3; Table 4). In the case of the roots, the transcriptional activity of these genes is high in Mcol2215 in relation to 60444, but sur- 
prisingly similar to the levels in Mtai16 (Figure 3; Table 4). Thus, apparently there is not a clear gene expression pattern according with the behavior waited. Nevertheless in this tissue, the ratio Mcol2215:Mtai16 is 3.44 times for linamarase, meanwhile the same ratio is 1.36 times for HNL. This indicates that in the sweet cultivar, the linamarse gene has high transcriptional activity in relationship with the bitter cultivar. The non statistical differences between these cultivars could be due to the high standard error in this experiment, as well as the low sample size used. This agrees with the previous studies conducted on linamarase by Santana et al. [9] where they found that linamarase activity from the root peel of a low cyanide cultivar is about 10 times higher than in the root peel of a high cyanide cultivar. Finally, in the case of the genes involved in the re-assimilation steps (like $\beta$-CAS), also we could think that the transcriptional activity is high in the sweet cultivar, reducing in this way the accumulation of cyanogenic glycosides. Our results are not according with this assumption, since that in leaves Mtai16 has higher $\beta$-CAS activity in relation to the other two cultivars, whereas in the roots was not observed any kind of differences. Thus, though traditionally the cultivar differences based on cyanogens are explained by the cyanogenic glycosides amounts present in the roots, we suggest that the difference in the cyanogenic glucosides in the roots are due to the combinatorial effect of their synthesis (CYP79D1/D2) in the leaves, their break-down in the roots by the linamarase, and the translocation of linamarin from leaves to roots. Furthermore, other aspects influencing the cyanogenic glucoside levels is the environment in combination with the developmental stage of the plant $[8,9]$.

Also, in the analysis of the expression of these genes between cultivars, interestingly, Mtail6 leaves had the highest expression levels compared to the other two cultivars. Meanwhile Mcol2215 and TMS60444 had very similar expression levels in the leaves. Such pattern was not observed in the roots. This pattern in the leaves of the three cultivars is correlated with the total HCN levels in each cultivar, where the amount of HCN between Mcol2215 and TMS60444 were more similar (142 ppm and $182 \mathrm{ppm}$, respectively) than Mtai16 amount (569 $\mathrm{ppm})$. Thus, bitter cassava is more dangerous for a herbivore not only due to the high levels of linamarin present but also for the high level of catabolic enzymes present that facilitate the faster release HCN when the foliar tissues are ingested by the animal. This also implies that although the synthesis, catabolism by disruption and the re-assimilation of HCN are spatially different and temporally separated events, they are coordinated well in the leaves. Such pattern was not observed in the roots between the three cultivars analyzed.
A multivariable approach in the data treatment was made, differing from the previous analysis in which univariable comparisons, in order to compare the transcriptional activity of all the genes (Figure 4). Here we can appreciate the lowest levels in roots of CYP79D1/D2 in relationship with the other genes. This expression profile, again reinforce the importance of the linamarin translocation for explain the presence of cyanogenic glycosides in the roots. Likewise, it is appreciated that the gene $\beta$-CAS, unlike to the other genes, did not show dramatic changes in the transcriptional activity between tissues. This result indicates the importance of the re-assimilation as a detoxification step; although also it can indicates the minor importance of these steps in the cultivars difference. Furthermore it is appreciated in the leaves a highest level of expression of HNL and linamarase, as well as similar level the expression of CYP79D1/D2 and $\beta$-CAS. These genes behaviors could indicate a co-regulation pattern. Thus, In order to find and verify this kind of pattern among the genes and among the cultivars, multivariable analysis with cluster analysis was performed. Comparisons were conducted for leaves and roots together per cultivar. A separation between HNL/linamarase and CYP79D1/D2/ $\beta$-CAS suggests a co-regulation between the catabolic cluster and the synthesis and re-assimilation cluster (Figure 5). These results are supported by the spatial separation in the cell wall of the HNL enzyme and linamarase enzyme, implying the presence of co-regulated due to their involvement in the break-down of cyanogens under plant disruption. It is important to consider that this clustering occurs in Mcol2215 and 60444 but not in Mtai16. The reason for this is the higher HNL level in the Mthai16 leaves (Figure 4). Another reason for the different cluster formation in Mtail6 could be due to the cyanide levels present in each cultivar, where Mcol2215 and 60444 are more equal compared to Mtai16. This is further supported by the clustering of Mcol2215 and TMS60444 into one group distant from Mtai16 (Figure 6).

This is the first report that shows quantitative and statistically significant differential expression of four genes involved in the cyanogenic glucoside pathway of cassava. The comparative analysis conducted between two tissues in three cassava cultivars, having different cyanogenic glucoside levels, shows the complexity that exists in the coordinated expression of these genes. In order to obtain more significant difference between cultivars or tissue it is important to increase the sample size thus reducing the high standard deviations present. With the platform established for the expression of these genes under normal conditions it is now possible to mimic abiotic stresses under in vitro conditions (such as drought, cold, mineral deficiencies etc.) in order to understand regulation of 
these genes under stress in cassava. Such studies then can lead to more expansive gene expression studies conducted with plants grown in the field, which are affected synergistically by abiotic and biotic stresses as well as by the development stage of the plant.

\section{Acknowledgements}

The authors would like to first and foremost thank Dr. Raul Macchiavelli (Department of Agronomy and Soils, UPRM) for help with the statistical analysis in this manuscript and Dr. Carlos Rodriguez and Dr. Rafael Montalvo (Department of Biology, UPRM) for use of the ABI7300 real-time PCR machine. We would also like to thank International Center of Tropical Agriculture (CIAT, Colombia) for its collaboration in the initial steps of this project. This work was possible due to funds provided to DS from the RIG/CAA program of the National Science Foundation. This manuscript is dedicated to Ida Munoz, a true friend of DS, who unfortunately passed away during the initial preparation of this manuscript.

\section{REFERENCES}

[1] H. Ceballos, "La Yuca en Colombia y el Mundo: Nuevas Perspectivas Para un Cultivo Milenario," In: H. Ceballos and B. Ospina, Eds., La Yuca en el Tercer Milenio, CIAT., Cali, 2002, pp. 1-13.

[2] M. El-Sharkawy, "Cassava Biology and Physiology," Plant Molecular Biology, Vol. 56, No. 4, 2004, pp. 481-501. doi:10.1007/s11103-005-2270-7

[3] M. Mejia de Tafur, "Fisiología de la yuca (Manihot esculenta Crantz)," In: H. Ceballos and B. Ospina, Eds., La Yuca en el Tercer Milenio, CIAT., Cali, 2002, pp. 34-45.

[4] J. McMahon, W. White and R. T. Sayre, "Cyanogenesis in Cassava (Manihot esculenta Crantz)," Journal of Experiment Botany, Vol. 46, No. 7, 1995, pp. 731-741. doi:10.1093/jxb/46.7.731

[5] W. White, D. Arias-Garzon, J. McMahon and R. T. Sayre, "Cyanogenesis in Cassava: The Role of Hydroxynitrile Lyase in Root Cyanide Production," Plant Physiology, Vol. 116, No. 4, 1998, pp. 1219-1225.

doi:10.1104/pp.116.4.1219

[6] K. Jørgensen, S. Bak, P. K. Busk, C. Sørensen, C. E. Olsen, J. Pounti-Kaerlas, B. L. Møller, "Cassava Plants with Depleted Cyanogenic Glucoside Content in Leaves and Tubers. Distribution of Cyanogenic Glucosides, Their Site of Synthesis and Transport, and Blockage of the Biosynthesis by RNA Interference Technology," Plant Physiology, Vol. 139, No. 1, 2005, pp. 363-374. doi:10.1104/pp.105.065904

[7] D. Siritunga and R. T. Sayre, "Engineering Cyanogen Synthesis and Turnover in Cassava (Manihot esculenta)," Plant Molecular Biology, Vol. 56, No. 4, 2004, pp. 661669. doi:10.1007/s11103-004-3415-9
[8] A. Cardoso, P. Ernesto, M. Cliff and J. H. Bradbury, "High Levels of Total Cyanogens in Cassava Flour Related to Drought in Mozambique," Roots, Vol. 6, 1999, pp. 4-6.

[9] M. A. Santana, V. Vasquez, J. Matehus and R. R. Aldao, "Linamarase Expression in Cassava Cultivars with Roots of Low- and High-Cyanide Content," Plant Physiology, Vol. 129, No. 4, 2002, pp. 1686-1694.

doi:10.1104/pp.000927

[10] O. Mkpong, H. Yan, G. Chism and R. T. Sayre, "Purification, Characterization, and Localization of Linamarase in Cassava," Plant Physiology, Vol. 93, No. 1, 1990, pp. 176- 181. doi:10.1104/pp.93.1.176

[11] M. D. Andersen, P. K. Busk, I. Svendsen and B. L. Møller, "Cytochromes P-450 from Cassava (Manihot esculenta Crantz) Catalyzing the First Steps in the Biosynthesis of the Cyanogenic Glucosides Linamarin and Lotaustralin," Journal of Biological Chemistry, Vol. 275, No. 3, 2000, pp. 1966-1975. doi:10.1074/jbc.275.3.1966

[12] M. A. Hughes, K. Brown, A. Pancoro, S. Murray, E .Oxtoby and J. Hughes, "A Molecular and Biochemistry Analysis of the Structure of the Cyanogenic $\beta$-Glucosidase (Linamarase) from Cassava (Manihot esculenta Crantz)," Archives of Biochemistry and Biophysics, Vol. 295, No. 2, 1992, pp. 273-279. doi:10.1016/0003-9861(92)90518-2

[13] J. Hughes, F. J. Carvallo, C. De and M. A. Hughes, "Purification, Characterization, and Cloning of Hydroxynitrile Lyase from Cassava (Manihot esculenta Crantz)," Archives of Biochemistry and Biophysics, Vol. 311, No. 2, 1994, pp. 496-502. doi:10.1006/abbi.1994.1267

[14] B. Koch, V. S. Nielsen, B. A. Halkier, C. E. Olsen and B. L. Moller, "The Biosynthesis of Cyanogenic Glucosides in Seedlings of Cassava (Manihot esculenta Crantz," Archives of Biochemistry and Biophysics, Vol. 292, No. 1, 1992, pp. 141-150. doi:10.1016/0003-9861(92)90062-2

[15] D. Selmar, R. Lieberei, B. Biehl and E. E. Conn, "Hevea Linamarase a Non Specific $\beta$-Glycosidase," Plant Physiology, Vol. 83, No. 3, 1989, pp. 557-63. doi: $10.1104 / \mathrm{pp} .83 .3 .557$

[16] D. Selmar, "Translocation of Cyanogenic Glucosides in Cassava," Acta Horticulturae, Vol. 375, 1994, pp. 61-67.

[17] D. Siritunga and R. T. Sayre, "Generation of CyanogenFree Transgenic Cassava,” Planta, Vol. 217, No. 3, 2003, pp. 367-373. doi:10.1007/s00425-003-1005-8

[18] F. Nartey, "Studies on Cassava Manihot utillisima, Biostnthesis of Asparagines- ${ }^{14} \mathrm{C}$ from ${ }^{14} \mathrm{C}$-Labelled Hydrogen Cyanide and Its Relations with Cyanogenesis," Physiologia Plantarum, Vol. 22, No. 5, 1969, pp. 10851096. doi:10.1111/j.1399-3054.1969.tb07470.x

[19] P. M. Dunhill and L. Fowden, "Enzymatic Formation of $\beta$-Cyanoalanine from Cyanide by Escherichia coli Extracts," Nature, Vol. 208, No. 5016, 1965, pp. 1206-1207. doi:10.1038/2081206a0

[20] H. G. Floss, L. Hadwiger and E. E. Conn, "Enzymatic Formation of $\beta$-Cyanoalanine from Cyanide," Nature, Vol. 208, 1965, No. 5016, pp. 1207-1208. 
doi: $10.1038 / 2081207 \mathrm{a} 0$

[21] P. A. Castric, K. J. Farnden and E. E. Conn, "Cyanide Metabolism in Higher Plants: The Formation of Asparagine from $\beta$-Cyanoalanine," Archives of Biochemistry and Biophysics, Vol. 152, No. 1, 1972, pp. 62-69. doi:10.1016/0003-9861(72)90193-2

[22] M. Makame, M. Akoroda and S. Hahn, "Effects of Reciprocal Stem Grafts on Cyanide Translocation in Cassava," Journal of Agricultural Sciences, Vol. 109, No. 3, 1987, pp. 605-608. doi:10.1017/S0021859600081855

[23] T. Ramanujam and P. Indira, "Effect of Girdling on the Distribution of Total Carbohydrates and Hydrocyanic Acid in Cassava," Indian Journal of Plant Physiology, Vol. 27, 1984, pp. 355-360.

[24] H. Dagher, H. Donninger, P. Hutchinson, R. Ghildyal and
P. Bardin, "Rhinovirus Detection: Comparison of RealTime and Conventional PCR," Journal of Virology Methods, Vol. 117, No. 2, 2004, pp. 113-121. doi:10.1016/j.jviromet.2004.01.003

[25] M. T. Dorak, "Real-Time PCR," In: M. T. Dorak, Ed., Real-Time PCR (Advanced Methods Series), Taylor \& Francis, Oxford, 2006, pp. 1-33.

[26] M. Elias, B. Nambisan and P. R. Sudhakaran, "Catabolism of Linamarin in Cassava," Plant Science, Vol. 126, No. 2, 1997, pp. 155-162. doi:10.1016/S0168-9452(97)00100-3

[27] M. Elias, P. Sudhakaran and B. Nambisan, "Purification and Characterization of $\beta$-Cyanoalanine Synthase from Cassava Tissues," Phytochemistry, Vol. 46, No. 3, 1997, pp. 469-472. doi:10.1016/S0031-9422(97)00305-1 